\title{
OR4-3
}

\section{Absence of tissue transglutaminase delays amyloid-beta deposition in Alzheimer's disease}

\author{
Micha M. M. Wilhelmus ${ }^{1}$, A B Smit', Maarten Loos ${ }^{3}$, Benjamin Drukarch ${ }^{1}$ \\ ${ }^{I}$ Anatomy and Neurosciences, VU University Medical Center, the Netherlands, ${ }^{2}$ Molecular and Cellular Neurobiology, \\ VU University Amsterdam, the Netherlands, ${ }^{3}$ Sylics (Synaptologics BV), Amsterdam, the Netherlands
}

Background: Alzheimer's disease (AD) is characterized by amyloid-beta $(\mathrm{Ab})$ aggregates in the brain as senile plaques and cerebral amyloid angiopathy. Targeting $\mathrm{Ab}$ aggregates has been one of the major approaches for $\mathrm{AD}$ therapies, although these attempts had little to no success so far. Therefore, novel treatment options should focus on blocking the actual formation of neurotoxic Ab multimers. Evidence is accumulating that tissue transglutaminase (tTG) plays a key role in these processes. TTG is abundantly expressed in human brain and catalyzes post-translational modifications resulting in covalently cross-linked protein complexes. $\mathrm{Ab}$ is a substrate for tTG cross-linking, resulting in stable and neurotoxic $\mathrm{Ab}$ oligomers. As such, tTG activity plays a prominent role in initiating the Ab cascade in AD. Therefore, in vivo absence of tTG in the AD-mimicking mouse model APP23 may provide evidence that $\mathrm{tTG}$ is a suitable target in AD to counteract $\mathrm{Ab}$ neurotoxicity.

Methods

We used a crossbreed of the tTG-/- mouse model and the AD-mimicking APP23 mouse model, and performed the following analysis: 1)(immuno)histochemical analysis and quantification of presence and severity of Ab pathology, 2) mRNA levels of APP and all brain TGs, and 3) Ab (1-40, 1-42 and ratio) protein analysis.

Results

We found that absence of tTG in 12-month-old APP23/tTG-/- mice resulted in an overall reduction of Ab deposits, whereas this difference was undetectable in 18-month-old animals. Analysis of the individual Ab deposits revealed that absence of tTG significantly reduced the formation of amyloid plaques, small dense plaques and vascular amyloid deposits when compared to 12-month-old APP23 mice. This significant difference was undetectable in the 18-month-old mice groups. In addition, we found an age-related increase in tTG mRNA levels in both APP23 and WT animals, whereas no compensation in mRNA levels of other transglutaminases for the absence of TG2 in the KO groups. Finally, we found a significant decrease in both Ab1-40 and Ab1-42 brain levels in APP23/tTG-/- mice when compared to APP23 mice.

Conclusion

Absence of tTG delays Ab pathology in the AD-mimicking APP23 mouse model. Therefore, tTG might be a suitable therapeutic target for reducing and/or delaying Ab deposition in $\mathrm{AD}$. 